\title{
Numerical Prediction of Hydromechanical Behaviour of Controllable Pitch Propeller
}

\author{
Saman Tarbiat, Hassan Ghassemi, and Manouchehr Fadavie \\ Department of Ocean Engineering, Amirkabir University of Technology, Tehran, Iran \\ Correspondence should be addressed to Hassan Ghassemi; gasemi@aut.ac.ir
}

Received 14 January 2014; Revised 10 April 2014; Accepted 14 April 2014; Published 9 July 2014

Academic Editor: Sergio Preidikman

Copyright (C) 2014 Saman Tarbiat et al. This is an open access article distributed under the Creative Commons Attribution License, which permits unrestricted use, distribution, and reproduction in any medium, provided the original work is properly cited.

\begin{abstract}
The research described in this paper was carried out to predict hydrodynamic and frictional forces of controllable pitch propeller (CPP) that bring about fretting problems in a blade bearing. The governing equations are Reynolds-averaged Navier-Stokes (RANS) and are solved by OpenFOAM solver for hydrodynamic forces behind the ship's wake. Frictional forces are calculated by practical mechanical formulae. Different advance velocities with constant rotational speed for blades are used to achieve hydrodynamic coefficients in open water and the wake behind the propeller. Results are compared at four different pitches. Detailed numerical results of 3D modelling of the propeller, hydrodynamic characteristics, and probability of the fretting motion in the propeller are presented. Results show that the probability of the fretting movement is related to the pitch.
\end{abstract}

\section{Introduction}

The main goal of any propulsion system is to obtain the required speed of a vessel. One way to increase the control, manoeuvrability, and efficiency is to utilize controllable pitch propellers instead of constant pitch ones. Specific characteristics of controllable propellers permit the blades to rotate around the propeller axle. Blades rotate with complex mechanism which acts mechanically or hydraulically. The ability of the CPP is to adjust the pitch in order to avoid the motor to be overloaded and lead to a decrease in shaft speed as well as to increase the motor duration. During recent years numerous investigations have been carried out on propeller designs. Consequently different methods have been presented.

Because of different application of propellers numerous layouts of propeller have emerged. Today CPP is used especially on vessels which operate at various operation conditions such as containers, fishing vessels, and towing vessels. For the first time in $1840 \mathrm{CPP}$ was introduced. At that time practical usage of CPP was in gas turbines. In twentieth century when diesel was introduced, usage of CPP increased suddenly. At that time pitch control was simple and mechanical. Dutch Schooner was the first one who applied CPP for diesels. In 1934, Escher Wyss used hydraulic mechanism in CPP which can be used for larger vessels. In 1937, Kamewa propellers were introduced. The hub mechanism of the mentioned propellers was based on Kaplan turbine design $[1,2]$.

In recent years numerous researches have been conducted on mechanism and the controlling system of CPP and on the ways to decrease the friction resistance and vibrations of the blades. Tendency to increase both hydrodynamic forces in CPP and usage of hydraulic systems for pitch adjustment leads to more investigations on CPP. For example, in 2005, Bakker studied different control strategies. He introduced mechanical systems of CPP propellers in detail [3]. In 2006, Dallinga calculated the forces acting on the CPP in different sea conditions [4]. In 2009, Stuart et al. calculated the forces acting on blades under cavitation condition [5]. A wake model for the prediction of propeller performance at low advance ratios was investigated by Tian and Kinnas [6]. In 2009, Godjevac et al. [7] focused on the forces acting in a controllable pitch propeller, mechanism, and the manifestation of vibrating motion (fretting) in a blade bearing caused by seaway.

Fretting is a special wear process which happens in contact surfaces which have a relative oscillatory motion of small amplitude, typically smaller than $1 \mathrm{~mm}$. Usually, the motion is caused by vibrations of the machinery. The fretting wear can occur at the CPP of the contact between blades and hub due 

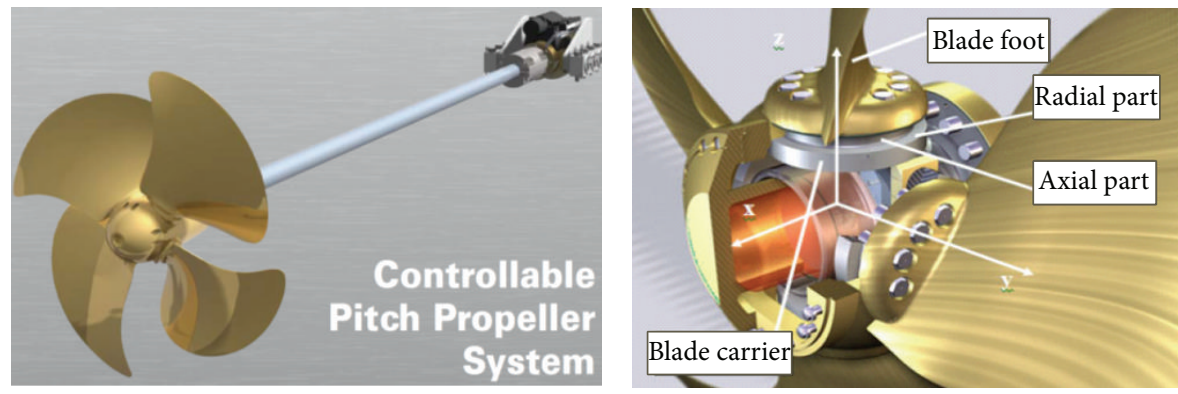

FIGURE 1: CPP configuration and its coordinate system [8].

to variable hydrodynamic loading acting on the blades. The critical moment for the commencement of fretting motion is when the spindle torque conquers the friction torque. As the thrust and torque of a blade change during one revolution, the Coulomb friction in the blade bearing alters as well during one revolution. Looking at the Coulomb friction in the blade bearing and spindle torque of the corresponding blade, it is possible to illustrate the occurrence of fretting motion. Figure 1 shows the CPP configurations and its coordinate system. Blade bearing is the bearing that connects the blade foot, blade carrier, and hub. Blade bearing has the radial and axial parts. Radial part of blade bearing has the inner side (closer to $x$-axis) and outer side [8].

The $x$-, $y$-, and $z$-axes are used to explain the forces and moments acting in a blade bearing. It also shows the radial and axial part of the blade bearing. The blade foot and the blade carrier are fastened with bolts. The critical moment for the beginning of fretting motion is when the spindle torque overcomes the friction torque. As the thrust and torque of a blade change during one revolution, the friction force in the blade bearing changes as well during one revolution. Looking at the friction force in the blade bearing and spindle torque of the corresponding blade, it is possible to illustrate the happening of fretting motion.

The efficiency of the propulsion system is strongly dependent on propeller performance, thrust force, torque of propeller, and its efficiency. Therefore this study focuses on the hydrodynamic performance and prediction of fretting motion in special CPP. The purpose of this study is to identify the effect of changes in pitch on propeller fretting motion.

Therefore, a simple method has been investigated to graph the propeller hydrodynamic coefficients with respect to advance coefficient and fretting occurring during one revolution of the blades. This investigation considers the situation that leads to fretting motion. When the hydrodynamic forces change during rotation of the blades, the friction in the blade bearings will change accordingly. By calculation of the friction in the blade bearing and spindle torque of the corresponding blade, it is possible to describe the occurrence of fretting motion.

\section{Three-Dimension Modelling of the Investigated Propeller}

$\mathrm{B}$-series propeller is a famous series which is used in almost all practical usages [1]. Pitch-diameter ratio in these series is

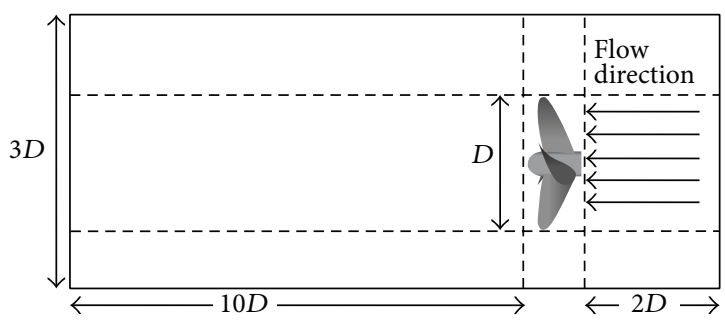

FIGURE 2: Numerical domain of propeller.

between 0.3 and 1.5. Number of the blades in B-series varies between 2 and 7. Usually four blades are used for merchant ships. Therefore, in our study four blades B-series propeller (B4-0.65) was simulated with the following characteristics:

$$
\begin{aligned}
& \text { number of blades }(Z): 4 \text {, } \\
& \text { diameter }(D): 0.508 \mathrm{~m}, \\
& \text { pitch ratio }(P / D): 0.5,0.8,1.1 \text {, and } 1.4 \text {, } \\
& \text { expanded area ration }\left(A_{E} / A_{0}\right): 0.65 \text {, } \\
& \text { rake angle }\left(\gamma_{r}\right): 15 \mathrm{deg} \text {., } \\
& \text { skew angle }\left(\gamma_{s}\right): 13.7 \mathrm{deg} .
\end{aligned}
$$

The experimental data of this propeller at open-water is available. First, we calculate the open-water characteristics and then extend to determine the hydrodynamic performance at nonuniform flow including frictional forces and looking for the fretting problem. To solve the fluid around the propeller, we used three parts: the stationary zone, moving zone, and the propeller zone.

Stationary Zone. This zone is cylindrical and includes the boss, propeller, and moving zone. We considered upstream length as $2 D$, downstream length is $10 D$, and the zone diameter is $3 D$, as shown in Figure 2 where $D$ is the diameter of the propeller.

\section{Numerical Procedure}

To investigate the hydrodynamic performance and flow fields around the propeller, Reynolds Navier-Stokes equations (RANS) for incompressible flow are applied. The governing equations are as follows. 


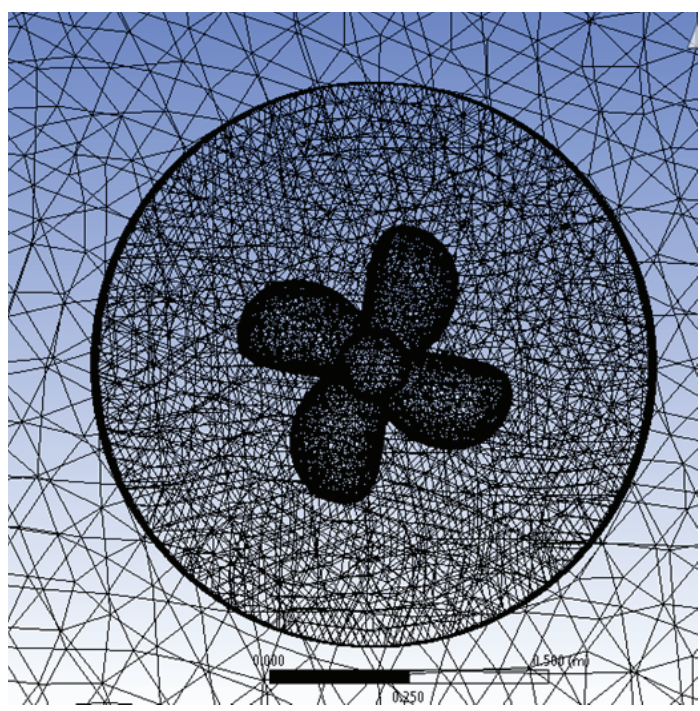

FIGURE 3: Final mesh for the calculation domain.

Conservation of mass (quantity equation):

$$
\frac{\partial \rho}{\partial t}+\frac{\partial}{\partial x_{j}}\left(\rho u_{j}\right)=0
$$

conservation of momentum:

$$
\begin{aligned}
& \frac{\partial \rho}{\partial t}\left(\rho u_{i}\right)+\frac{\partial}{\partial x_{j}}\left(\rho u_{i} u_{j}\right) \\
& \quad=\frac{\partial}{\partial x_{j}}\left[-p \delta_{i j}+\mu\left(\frac{\partial U_{i}}{\partial X_{j}}+\frac{\partial U_{J}}{\partial X_{i}}\right)\right]+B_{i},
\end{aligned}
$$

where $B_{i}=$ body force.

Unstructured triangle cells are used to describe the control volume. By using the momentum, conservation of mass and energy equation around the propeller and the mathematical condition imposed at solid and fluid boundaries. Governing equations are solved. We should consider the time and cost in calculation. We used different number of meshes to reach its independency for the final results of propeller characteristics $\left(K_{T}, K_{Q}\right.$, and $\left.\eta_{O}\right)$. Size of meshes near the hub, blade edges, and tip of the blades is smaller than other parts. The domain was meshed by tetrahedral cells as shown in Figure 3. Instead of rotating the propeller, fluid region around the propeller is rotated.

To ensure the grid independency, different grids numbers have been chosen [10]. It is found that with the number 880000 grids all parameters such as thrust coefficient are converged. The important values $y^{+}$are less than 50 for all advance velocity ratios $(J)$ when $K_{T}$ is converged. Gird number, thrust coefficient, and its $y^{+}$values are shown in Table 1.

\section{Hydrodynamic Performance of the Investigated Propeller}

The most important characteristics of the marine propellers are the hydrodynamic performances that are operated in
TABLE 1: Grid number, thrust coefficient, and its $y^{+}$values $(P / D=$ $0.8)$.

\begin{tabular}{lcc}
\hline Number of grids & Thrust coeff. $\left(K_{T}\right)$ & $y^{+}$ \\
\hline 400000 & 0.26 & 550 \\
600000 & 0.31 & 190 \\
800000 & 0.35 & 30 \\
880000 & 0.352 & 25 \\
\hline
\end{tabular}

open-water flow. The hydrodynamic open-water characteristics are expressed as follows:

$$
K_{T}=\frac{T}{\rho n^{2} D^{4}}, \quad K_{\mathrm{Q}}=\frac{\mathrm{Q}}{\rho n^{2} D^{5}}, \quad \eta_{\mathrm{O}}=\frac{K_{T}}{K_{\mathrm{Q}}} \frac{J}{2 \pi},
$$

where $T$ and $Q$ are the thrust and torque of the propeller, respectively. $J$ is the advance velocity ratio and, defined by $J=$ $V_{A} / n D$, means advance velocity $\left(V_{A}\right)$ divided by rotational speed $(n)$ and diameter $(D)$.

Here, we selected that the rotational speed of the propeller is constant and equal to $120 \mathrm{rpm}$ and diameter is 0.508 [m]. The advance velocity is changed in order to calculate the propeller performance. The hydrodynamics characteristics of the propeller are performed and compared at four pitchdiameter ratios, that is, $P / D=0.5,0.8,1.1$, and 1.4. Figures 4, 5,6 , and 7 show the comparison of the hydrodynamic coefficients. General trends of the results are good but there are some discrepancies at off-design conditions. The velocity and pressure contours around the propeller in $J=1$ are shown in Figures 8 and 9, respectively. As observed in Figure 8, the pressure shows at face side of the propeller. The pressure is different on each blade due to nonuniform wake flow.

\section{Friction Forces}

CPP mechanism is much complicated because the propeller should utilize the engine power to obtain the optimum efficiency at all conditions. Propeller operates behind the ship hull where the flow is nonuniform and oscillating flow. Thus, the resultant velocity will change during one cycle and as a result the thrust will be oscillated. For this reason the propeller pitch should be adjusted based on the required thrust loading. Therefore, the blade pitch changes at any time during one revolution. The blade seat on the hub is made by a bearing that supports forces in both axial and radial directions, as shown in Figure 10.

The friction forces due to controlling the blade on the hub bearing are divided into two axial and radial directions. The forces and moments have been evaluated by the procedure proposed [7]. The resulting forces in the axial part of the blade are defined through

$$
\begin{aligned}
F_{a x} & =\sqrt{F_{a x, x}^{2}+F_{a x, y}^{2}}, \\
F_{a x, x} & =F_{\mathrm{hd}, x}, \\
F_{a x, y} & =F_{\mathrm{hd}, y}+F_{\mathrm{ce}, y},
\end{aligned}
$$




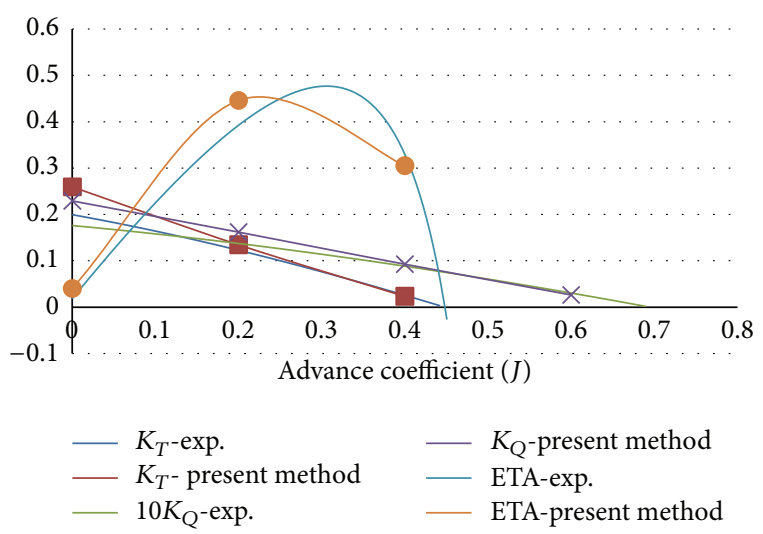

FIGURE 4: Hydrodynamic coefficient of the investigated propeller with $P / D=0.5$.

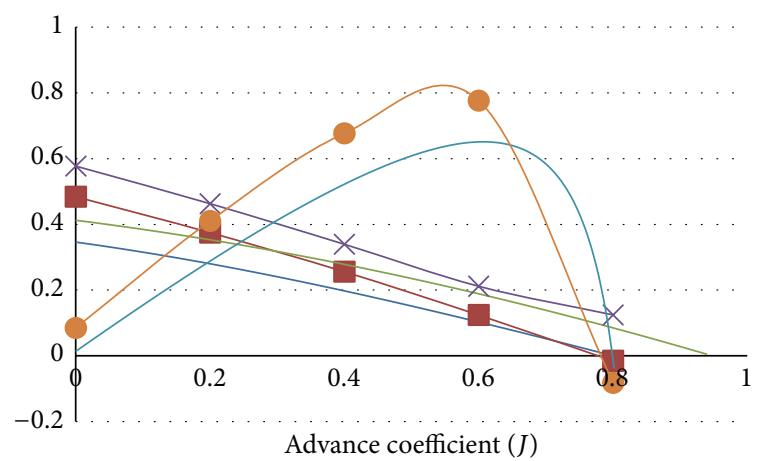
$\begin{array}{ll}- & K_{T^{-}} \text {exp. } \\ - & K_{T^{-}} \text {present method } \\ - & 10 K_{Q^{-}} \text {exp. }\end{array}$
- $K_{Q^{-} \text {-present method }}$
- ETA-exp.
— ETA-present method

FIGURE 5: Hydrodynamic coefficient of the investigated propeller with $P / D=0.8$.

where $F_{\mathrm{hd}, x}$ and $F_{\mathrm{hd}, y}$ are the components of the hydrodynamic forces, means $F_{\mathrm{hd}, x}=T$ (Thrust), $F_{\mathrm{hd}, y}=\mathrm{Q}$ (Torque) $/ r_{\mathrm{Q}}$. $r_{\mathrm{Q}}$ means the radius of centroid of torque. $F_{\mathrm{ce}, y}$ is the component of centrifugal forces. The axial torque due to the axial force is given by

$$
\mathrm{Q}_{\mathrm{Fr}, a x}=k_{f} \cdot F_{a x} \cdot d_{a},
$$

where $d_{a}$ is the distance between the point where the axial force is supposed to be applied and the hub friction seat of the propeller blade root. $k_{f}$ is the nondimensional frictional coefficient of the blade and hub materials. These parameters are given by the following formulae [2]:

$$
\begin{aligned}
k_{f}= & \frac{\mu}{\sqrt{1+\mu^{2}}} \\
d_{a}= & r\left(1+0.0477 \sin ^{2} \alpha+0.544 \sin ^{4} \alpha\right. \\
& \left.\quad-1.051 \sin ^{6} \alpha+0.6982 \sin ^{8} \alpha\right) .
\end{aligned}
$$

$r$ is the radius of blade bearing. The angle $\alpha$ depends on the axial force, friction coefficient, radius of blade bearing and

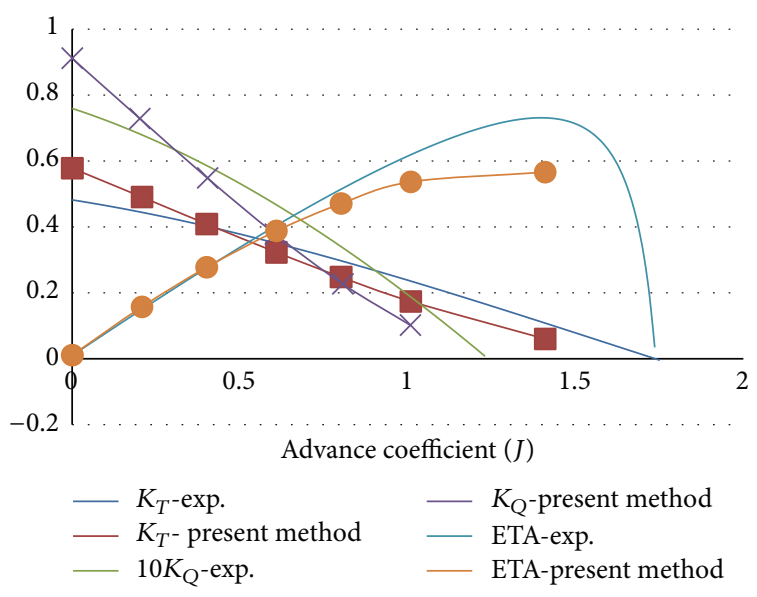

FIGURE 6: Hydrodynamic coefficient of the investigated propeller with $P / D=1.1$.

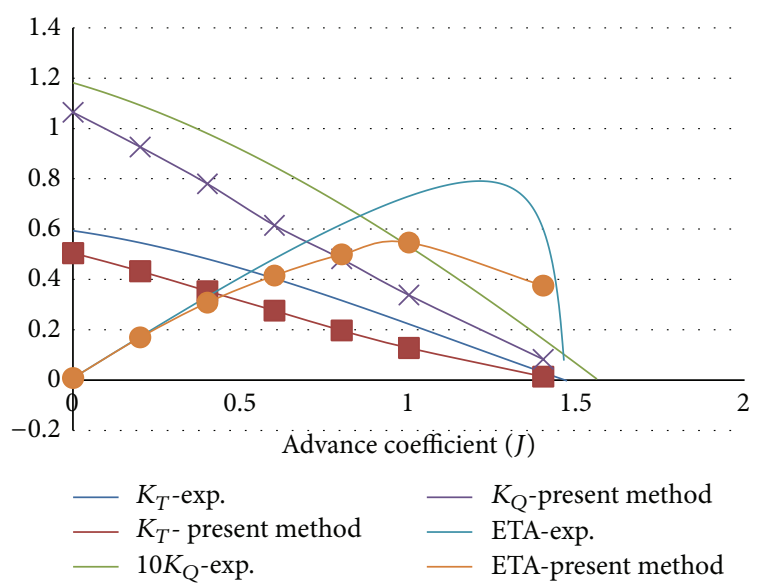

FIGURE 7: Hydrodynamic coefficient of the investigated propeller with $P / D=1.4$.

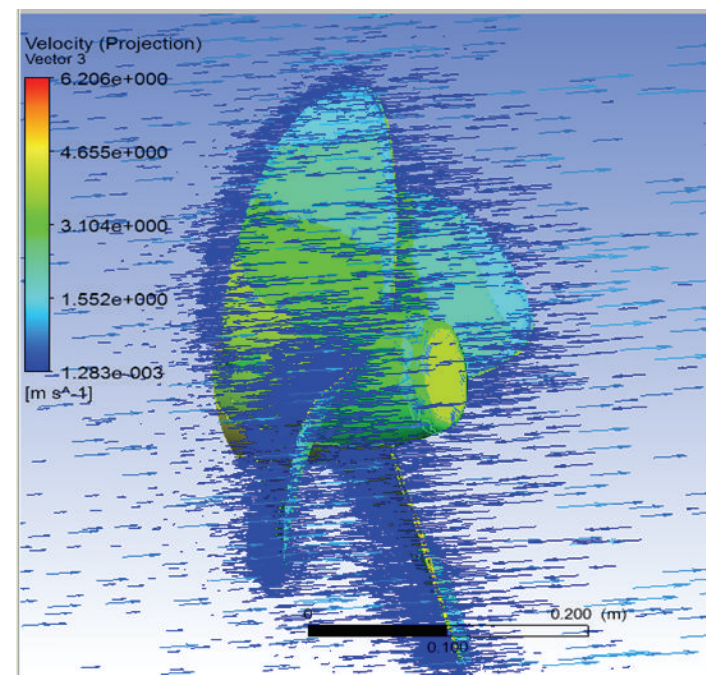

Figure 8: Contours of velocity $(J=1)$. 


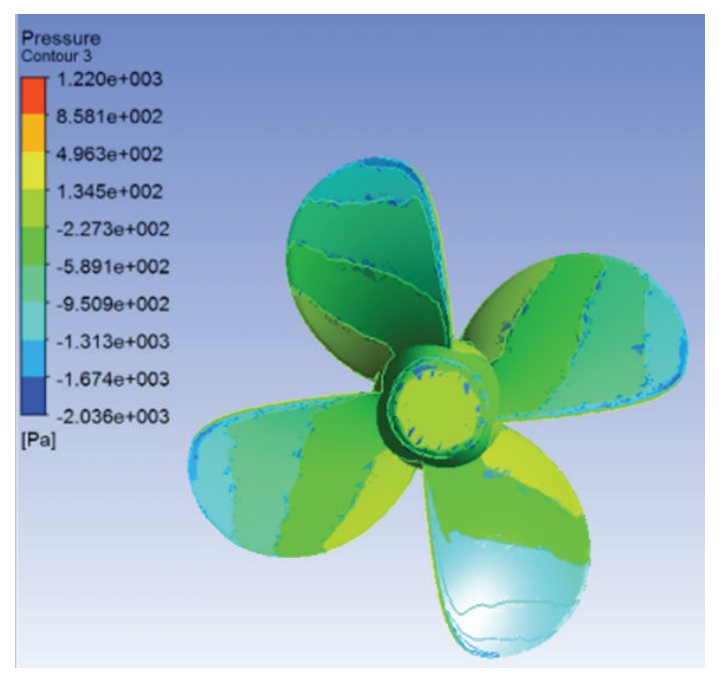

Figure 9: Pressure contour on suction surface $(J=1)$.

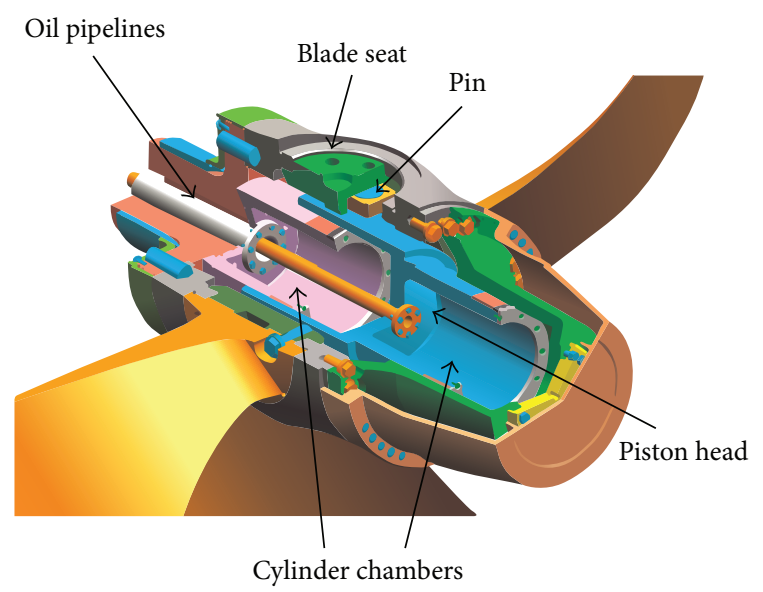

(a)

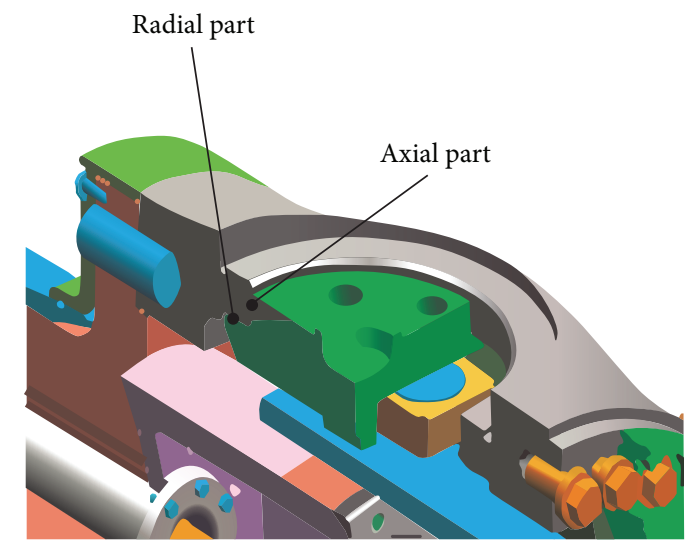

(b)

FIgUre 10: Main element inside a CPP hub (a). Blade seat (b) [9].

radius of blade carrier, thickness of blade bearing, and Young's modulus of elasticity. Here, the value of friction coefficient $(\mu)$ is about 0.1 and $\alpha$ is approximately 170 [deg.] and they are chosen for present calculations.
In the radial part the moments are given by

$$
\begin{aligned}
M_{a x} & =\sqrt{M_{a x, x}^{2}+M_{a x, y}^{2},} \\
M_{a x, x} & =-M_{\mathrm{hd}, x}, \\
M_{a x, y} & =M_{\mathrm{hd}, y}+M_{\mathrm{ce}, y},
\end{aligned}
$$

where $M_{\mathrm{hd}, x}$ and $M_{\mathrm{hd}, y}$ are the components of the hydrodynamic moments and $M_{c e, y}$ is the component of centrifugal moments. The hydrodynamic moments acting on the centre of hydrodynamic $(\mathrm{CH})$ of a $\mathrm{CPP}$ blade are calculated by

$$
\begin{aligned}
& M_{\mathrm{hd}, x}=\left(\frac{\mathrm{Q}}{r_{\mathrm{Q}}}\right) \cdot z_{\mathrm{CH}}, \\
& M_{\mathrm{hd}, y}=T \cdot z_{\mathrm{CH}}, \\
& M_{\mathrm{hd}, z}=T \cdot y_{\mathrm{CH}}+\left(\frac{\mathrm{Q}}{r_{\mathrm{Q}}}\right) \cdot x_{\mathrm{CH}},
\end{aligned}
$$

where $\left(x_{\mathrm{CH}}, y_{\mathrm{CH}}, z_{\mathrm{CH}}\right)$ is the hydrodynamic force centre. Finally, the friction torque in the radial part of the blade bearing is expressed as [11]

$$
Q_{\mathrm{Fr}, \mathrm{rad}}=\frac{4}{\pi} \cdot \mu \cdot M_{a x}\left(\cos \Phi_{0}+u \cdot \arcsin (u)\right),
$$

where $u$ is a coefficient that describes the ratio of the radial force and the maximum force resulting from the bending moment and $\Phi_{0}$ is the angle of circumference around blade bearing. Total friction torque of the blade bearing is obtained by $Q_{\mathrm{Fr}}=\sqrt{Q_{\mathrm{Fr}, a x}^{2}+Q_{\mathrm{Fr}, \mathrm{rad}}^{2}}$.

The fretting motion will happen when the blade spindle torque becomes higher than the friction torque, which means $M_{\mathrm{hd}, z}>Q_{\mathrm{Fr}}$.

\section{Results and Discussion}

Fretting motion starts when the sum of the forces acting on the body overcomes the friction force. As mentioned, it may occur when the blade spindle torque becomes bigger than the friction torque. In order to investigate the start of the fretting motion in a CPP, the relation between friction torque and the spindle torque during one revolution was accomplished.

In this study, we focused on the effect of different pitches on fretting behaviour of the CPP. Propeller is working in behind the ship hull where the wake flow which is into the propeller is nonuniform and oscillating flow. Wake factor $(1-w)$ is defined by $V_{A} / V_{S}$, where $V_{A}$ is advance velocity behind the ship and $V_{S}$ is the speed of the ship. Due to oscillating wake filed, the hydrodynamic forces will change in the wake field behind the propeller. The investigated wake field $(1-w)$ during one revolution of the blades shows different radius of the blades in Figure 11. The results of the spindle torque coefficient for different pitches for one revolution are presented in Figure 12. The mean values of the hydrodynamic Forces are presented in nondimensional values in Table 2. 
TABLE 2: Hydrodynamic forces of the investigated propeller.

\begin{tabular}{lcc}
\hline$P / D$ & {$\left[F_{\mathrm{hd}, x}, F_{\mathrm{hd}, y}\right]$} & {$\left[M_{\mathrm{hd}, x}, M_{\mathrm{hd}, y}, M_{\mathrm{hd}, z}\right]$} \\
\hline 0.5 & {$[-29.84,5.36]$} & {$[0.68,3.82,0.38]$} \\
0.8 & {$[-51.45,12.82]$} & {$[1.65,6.64,0.60]$} \\
1.1 & {$[-71.93,23.83]$} & {$[3.09,9.35,0.90]$} \\
1.4 & {$[-89.72,72.34]$} & {$[9.38,11.64,1.55]$} \\
\hline
\end{tabular}

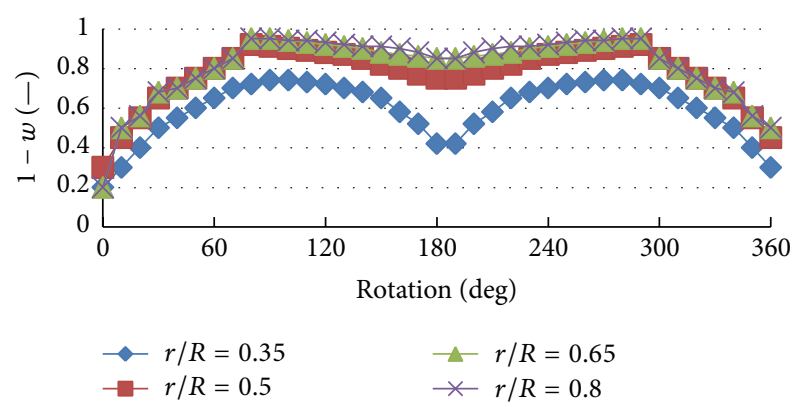

FIGURE 11: Nominal wake-field around the investigated propeller.

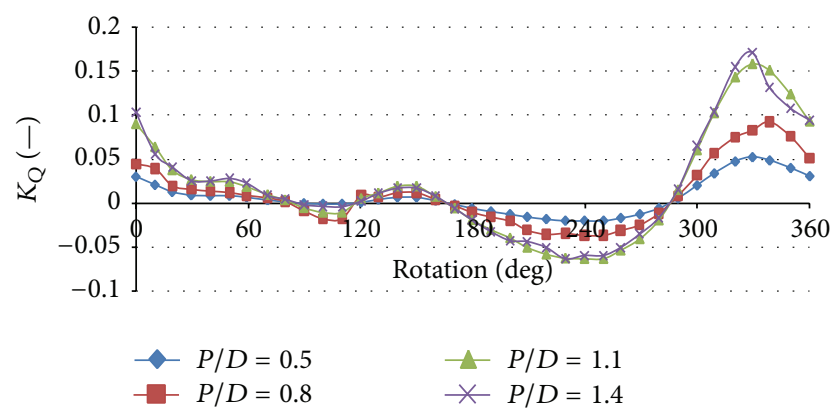

FIGURE 12: Spindle torque of the propeller during one revolution.

Friction forces are calculated based on given formulae. Figures 13, 14, 15, and 16 show the results of friction force and spindle torque during one revolution for different pitches $(0.5,0.8,1.1$, and 1.4$)$ of investigated propeller. It is obvious that the blue line which demonstrates the spindle torque is above the friction force (red line) in some cases. Looking at Figure 13, it is possible to determine the angle of rotation at which the spindle torque exceeds the friction torque. The spindle torque is larger than the friction torque between 119 and 132 degrees, and between 290 and 307 degrees of rotation. For the other pitch ratio of this propeller, fretting motion happen within other angles. Because the input of the wake flow into the propeller is the same for all geometrical pitch, the fretting occurred around 120 and 300 degrees. This is due to many changes of the torque at those angles.

\section{Conclusions and Future Work}

The numerical method based on RANS equations is applied to solve the hydrodynamic prediction of the propeller as well as frictional forces to show the fretting conditions. The hydrodynamic coefficient results in an open-water are relatively in agreement with experimental data. The results of the fretting

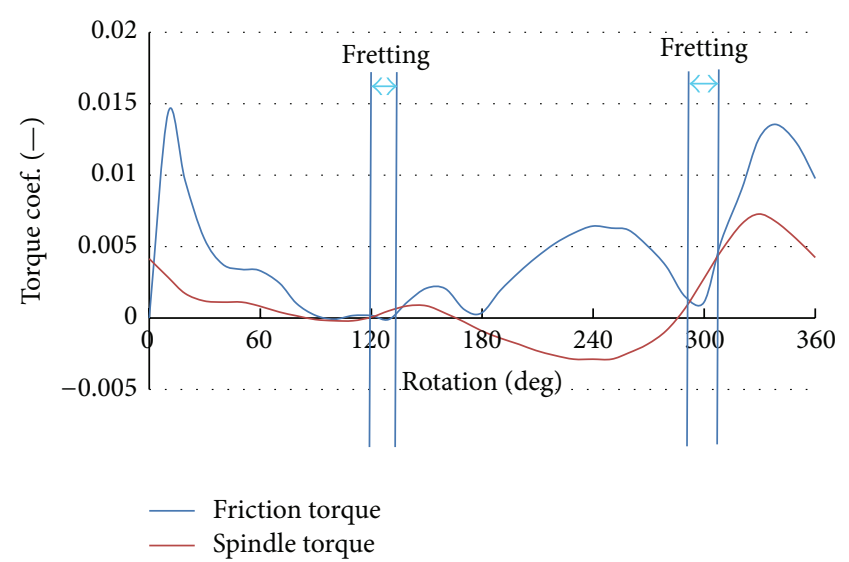

FIGURE 13: Fretting region of the propeller during one revolution in $P / D=0.5$.

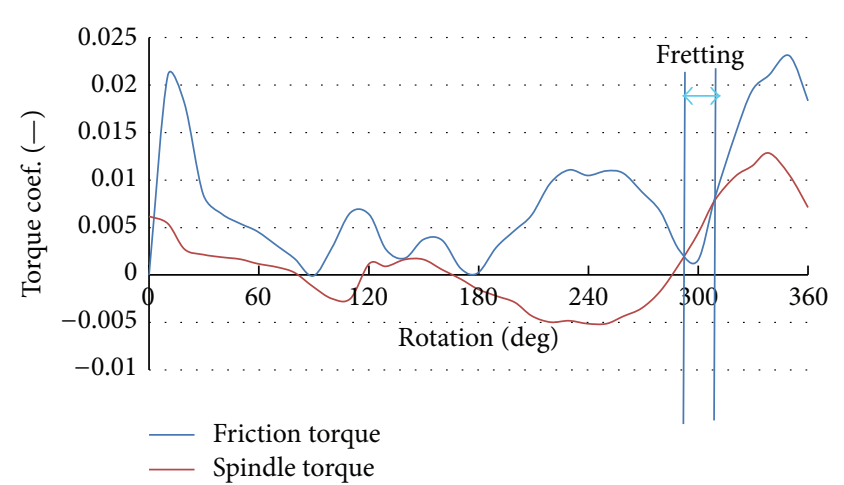

FIGURE 14: Fretting region of the propeller during one revolution in $P / D=0.8$.

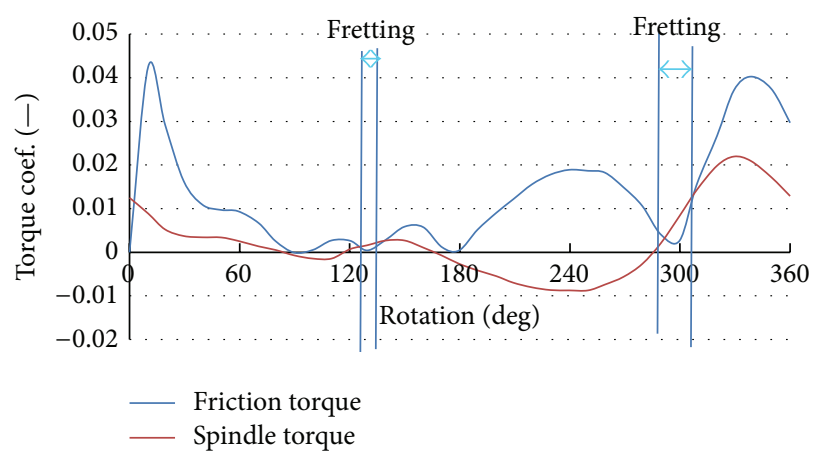

FIGURE 15: Fretting region of the propeller during one revolution in $P / D=1.1$.

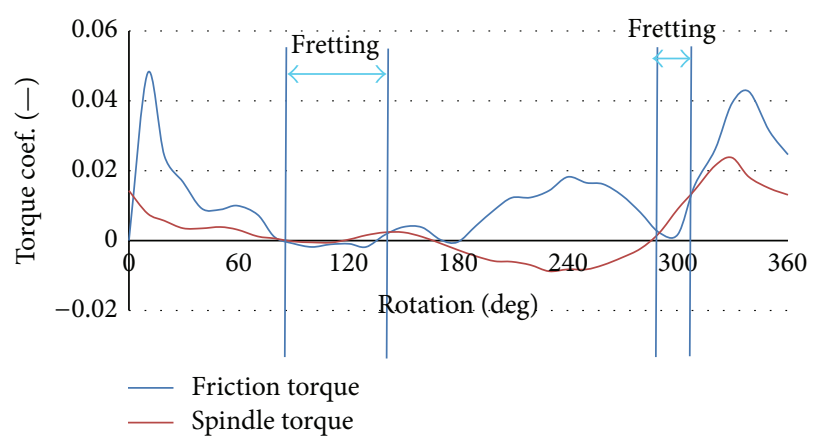

FIGURE 16: Fretting region of the propeller during one revolution in $P / D=1.4$. 
behaviour of the propeller at different pitches for the investigated propeller are presented. It shows that for the current propeller, when pitch ratio increases, the possibility of the fretting motion increases.

In the near future, an investigation will be carried out if the geometry of this or other propellers is made available. Also, effect of the ocean wave may be studied on the fretting problem. More accurate formulae can be achieved to determine the frictional forces on the blade bearing.

\section{Conflict of Interests}

The authors declare that there is no conflict of interests regarding the publication of this paper.

\section{Acknowledgments}

This research was supported by the High Performance Computing Research Center (HPCRC) at Amirkabir University of Technology, which is acknowledged. The authors wish to thank the reviewers for their valuable comments and suggestion.

\section{References}

[1] J. S. Carlton, Marine Propellers and Propulsion, ButterworthHeinemann, London, UK, 2nd edition, 2007.

[2] M. Martelli, M. Figari, and M. Altosole, "Controllable pitch propeller actuating mechanism, modeling and simulation," Journal of Engineering for the Maritime Environment, vol. 227, 2013.

[3] J. Bakker, Dynamic behaviour and nonlinear aspects of the CPP [M.S. thesis], TU Delft, Delft, The Netherlands, 2005.

[4] R. P. Dallinga, "Bow flare slamming of container ships and it's impact on operational reliability," in Proceedings of the Royal Institution of Naval Architects International Conference-Design and Operation of Container Ships (RINA '06), pp. 131-139, London, UK, November 2006.

[5] J. Stuart, M. Donnelly, and I. McClintock, "Measurements of controllable pitch propeller blade loads under cavitating conditions," in Proceedings of the 1st International Symposium on Marine Propulsors (SMP '09), Trondheim, Norway, June 2009.

[6] Y. Tian and S. A. Kinnas, "A wake model for the prediction of propeller performance at low advance ratios," International Journal of Rotating Machinery, vol. 2012, Article ID 372364, 11 pages, 2012.

[7] M. Godjevac, T. van Beek, H. T. Grimmelius, T. Tinga, and D. Stapersma, "Prediction of fretting motion in a controllable pitch propeller during service," Proceedings of the Institution of Mechanical Engineers, vol. 223, no. 4, pp. 541-560, 2009.

[8] http://www.wartsila.com/en/propulsors/propellers/CPP.

[9] http://www.rolls-royce.com/marine/products/propulsors/propellers/cpp/index.jsp.

[10] R. Shamsi and H. Ghassemi, "Numerical investigation of yaw angle effects on propulsive characteristics of podded propulsors," International Journal of Naval Architecture and Ocean Engineering, vol. 5, no. 2, pp. 287-301, 2013.

[11] A. Faraz and S. Payandeh, "Towards approximated models of Coulomb frictional moments," Journal of Engineering Mathematics, vol. 40, no. 3, pp. 283-296, 2001. 

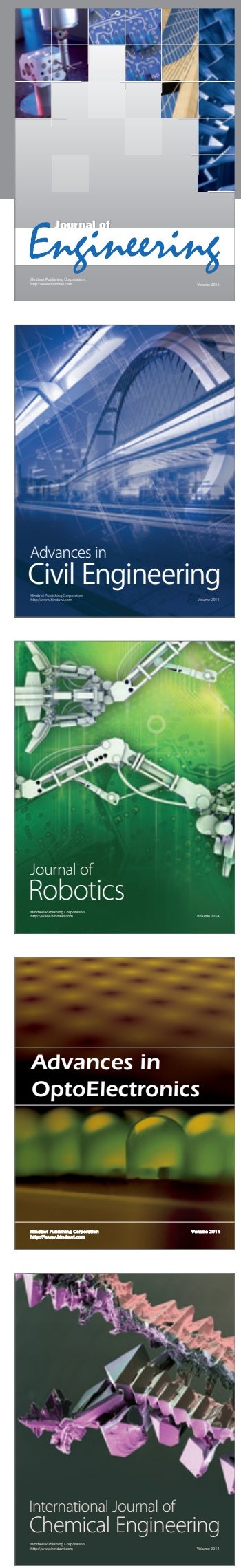

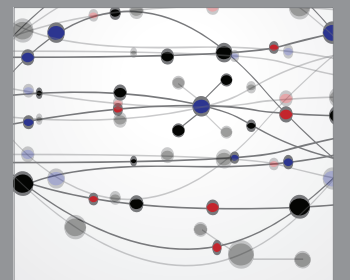

The Scientific World Journal
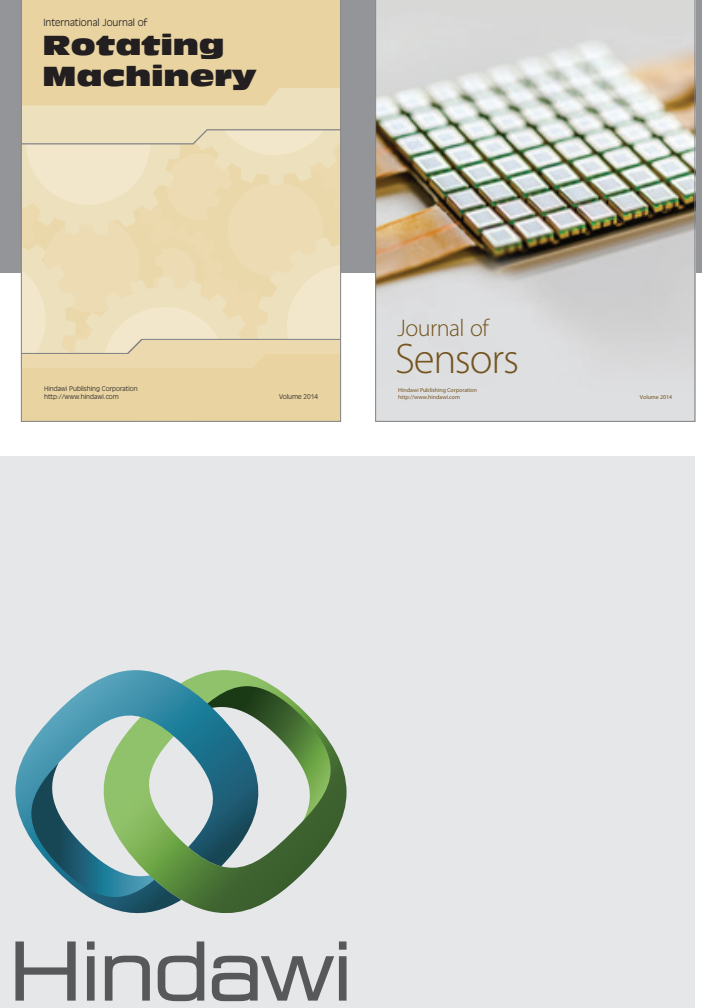

Submit your manuscripts at http://www.hindawi.com
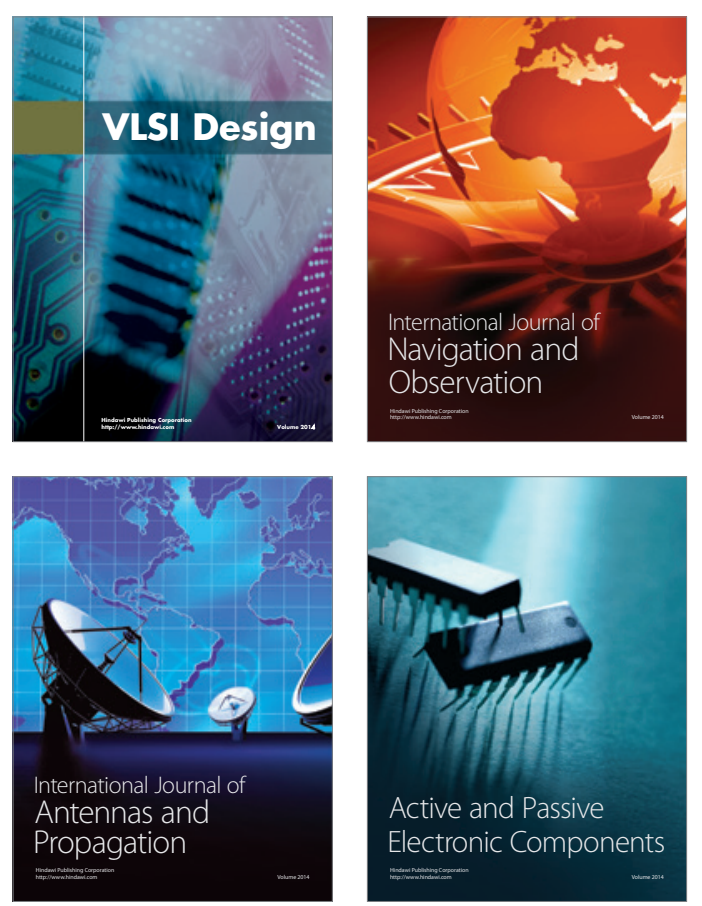
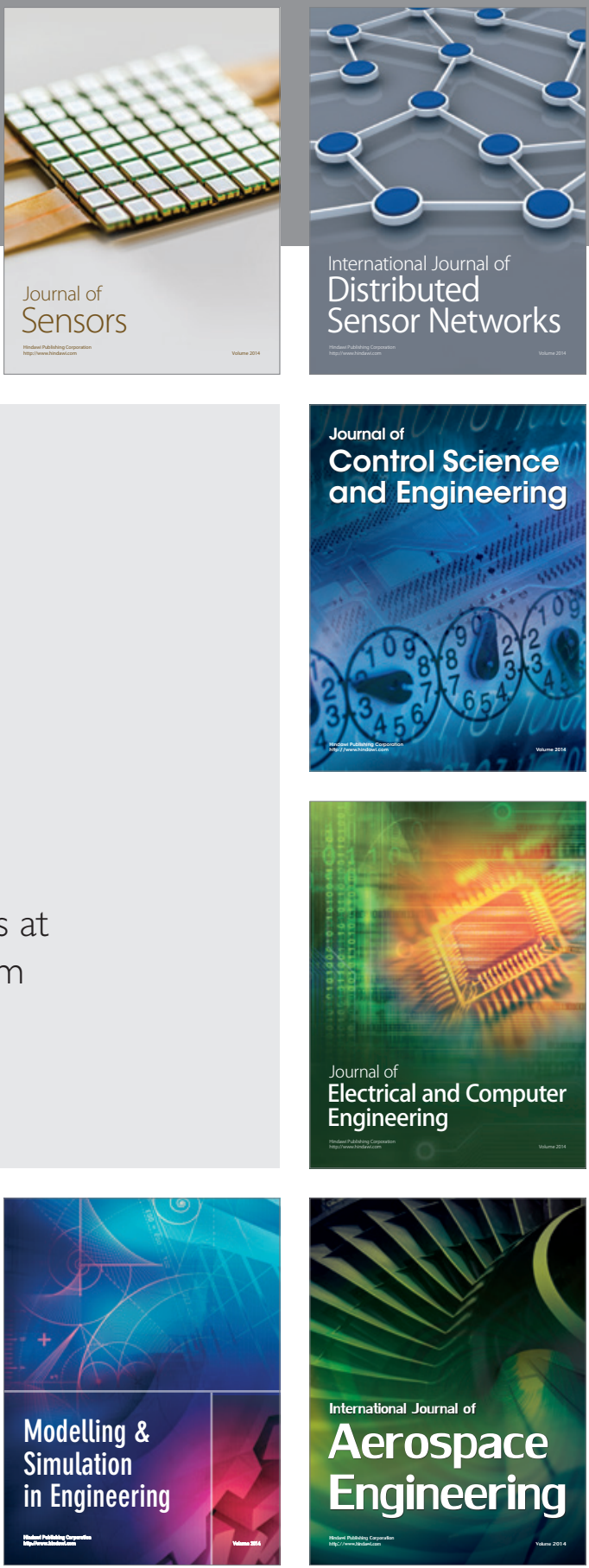

Journal of

Control Science

and Engineering
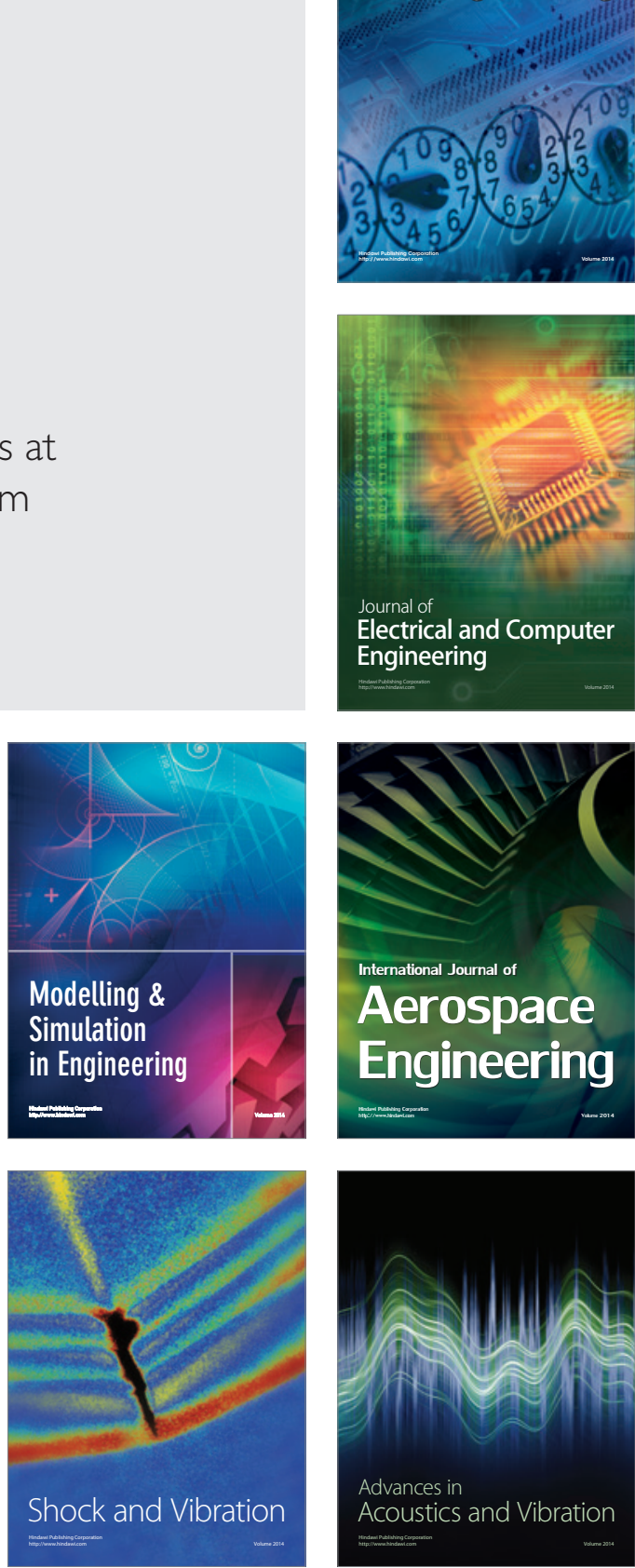